\title{
La caja de herramientas como opción metodológica ${ }^{1}$
}

\author{
Tomás Sánchez Amaya² \\ Universidad Santo Tomás-Bogotá
}

Recibido: 2 marzo de 2009 • Aprobado: 5 de marzo de 2010

\section{Resumen}

El presente artículo constituye un esbozo sobre la arqueología, en tanto herramienta metodológica, que proporciona recursos para abordar indagaciones sobre: temáticas, problematizaciones, discursos y objetos que yacen al margen de los grandes relatos, de las grandes líneas del pensamiento -de las cuales se ha ocupado la historia tradicional-. En perspectiva de poner en funcionamiento tal herramienta, el grupo de investigación Docimófilos ha diseñado una indagación que busca, a través de varias etapas, realizar una "caracterización de las formas de evaluación educativa en la Universidad Santo Tomás -Sede Bogotá, Modalidad Presencial-". Esta descripción histórica (arqueológica) no pretende desentrañar el origen primero y el fin último, el desarrollo evolutivo, la tradición y la autoridad del objeto propuesto (de la evaluación, sus discursos y prácticas), por cuanto determina las condiciones históricas por medio de las cuales se constituyen unos saberes específicos a partir de determinadas prácticas sociales.

Palabras clave: metodología, investigación, arqueología, genealogía, subjetividad, historización, analítica-histórica, evaluación, educación, examen.

1 Artículo de investigación que fundamenta metodológicamente la primera etapa de la indagación propuesta: "Caracterización de las Formas Evaluación Educativa en la Universidad Santo Tomás Sede Bogotá, Modalidad Presencial”.

2 Licenciado en Filosofía e Historia, Especialista en Educación y Filosofía Colombiana, Magíster en Filosofía Latinoamericana, Universidad Santo Tomás; Doctor en Ciencias Sociales, Niñez y Juventud, Universidad de Manizales-CINDE. Contacto: tas@etb.com.co. Hace parte del grupo de investigación Docimófilos (docimofilos@yahoo.es), avalado por el Departamento de Humanidades y Formación Integral y la Facultad de Comunicación Social de la USTA; inscrito en COLCIENCIAS, mediante el código colombiano de registro de grupos (CCRG) COL0069747, de noviembre de 2006. 


\section{The tool box as a methodology option}

\section{Abstract}

This article is an outline on the archeology, as a methodological tool, which provides resources to address inquiries about: topics, problems, speeches and objects that lie outside of the big stories of the great lines of thought, of which traditional history has studied. In view of putting into operation such a tool the research group Docimófilos has designed an inquiry looking through several stages, to make a characterization of the educational evaluation forms in Santo Tomás University in Bogotá, presencial mode. "This description historical (archaeological) is not designed to unravel the first origin and final end, the evolutionary development, tradition and authority of the proposed object (of the assessment, their discourses and practices), as determined by the historical conditions of which are a shared knowledge of certain social practices.

Key words: methodology, research, archeology, genealogy, subjectivity, historicizing, historical analytical, evaluation, education, examination. 


\section{La boîte à outils comme option méthodologique}

\section{ésumé}

Le présent article constitue une ébauche sur l'archéologie, comme outil méthodologique, qui offre des ressources pour aborder des investigations sur des thématiques, problématisations, discours et objets qui surgissent en marge des grands récits, des grandes lignes de la pensée dont s'est occupée I'histoire traditionnelle. Dans la perspective de la mise en fonction d'un tel outil, le groupe de recherche Docimófilos a dessiné une recherche qui tente, à travers plusieurs étapes, de réaliser une "caractérisation des formes d'évaluation éducatives à I'Université Santo Tomas, siège de Bogotá, modalité présentielle". Cette description historique (archéologique) ne prétend pas éclaircir l'origine première et la fin ultime, le développement évolutif, la tradition et l'autorité de l'objet proposé (l'évaluation, ses discours et ses pratiques) mais déterminer les conditions historiques au moyen desquelles se constituent des savoirs spécifiques à partir de pratiques sociales déterminées.

Mots-clé: méthodologie, recherche, archéologie, généalogie, subjectivité, historisation, analyse historique, évaluation, éducation, examen. 


\section{ntroducción}

Emergente de diversos intereses -personales e institucionales-, el grupo de investigación Docimófilos, una vez constituido, realizó una apuesta (propuesta investigativa) referida al estudio histórico (arqueológico-genealógico) de la evaluación en la Universidad Santo Tomás (USTA), sede Bogotá, en la modalidad presencial, con una triple intencionalidad: en primera instancia, caracterizar e historiar las modalidades de evaluación -discursos, prácticas, formas específicas de operar- que han hecho presencia en la Institución desde su refundación hasta el presente; posteriormente, enunciadas las formas evaluativas ${ }^{3}$, proceder a determinar, mediante una analítica-histórica, las condiciones de posibilidad que le han permitido su emergencia y su instalación a través de todo el conjunto de las prácticas institucionales y las formas de proceder allí, en tanto trama de relaciones de poder; finalmente, con la mediación de una analítica interpretativa, determinar tipologías de sujetos -y objetos- que son moldeados, modelados, formateados, agenciados, fabricados..., a través de las prácticas panevaluativas. Cada una de estas intencionalidades comporta, en cuanto tal, una etapa del proceso investigativo: una descripción arqueológica, una reconstrucción genealógica y una analítica de la subjetividad; todo ello referido a la evaluación educativa en la USTA.

Con la perspectiva de concretar las intencionalidades referidas, el equipo determina como instrumental metodológico el uso de la caja de herramientas, desarrollos teóricos foucaultianos que, como dispositivos de investigación, permiten el abordaje de historias particulares, de temáticas marginales, de cuestiones que por sus condiciones (históricas, sociales, ideológicas...) escapan a los análisis de las grandes teorías y de discursos científicos; porque en las más de las veces permanecen ocultas, quizá por hallarse un poco más allá de lo evidente, de lo visible, de lo perceptible, etc.

3 Por formas evaluativas entendemos: las diversas modalidades de prácticas evaluativas, el conjunto de posibilidades, la multiplicidad de modos, formas y estilos, en que la evaluación opera, en unas condiciones y circunstancias concretas, en un contexto histórico-social determinado; prácticas evaluativas que implican un conjunto de relaciones constituidas por instituciones, sujetos, teorías, prácticas, que de múltiples maneras han permeado el ser y el acontecer de la vida actual. El análisis de tales formas de evaluación educativa se realiza, según se ha enunciado, en ámbitos específicos (el nivel de educación superior), en instancias concretas (la Universidad Santo Tomás, sede Bogotá, modalidad presencial) y en una periodización determinada, desde su refundación (1965) hasta el presente. 
Así las cuestiones, la indagación propuesta busca recrear y actualizar en un ámbito de saber (y de poder) específico -el de la evaluación-, una arqueología, con la perspectiva de indagar sobre las formas de problematización en las que la verdad se formula, y una genealogía, con el objeto de analizar las prácticas por las que las propias problematizaciones se generan y modifican:

las problematizaciones a cuyo través el ser se da como poderse y deberse ser pensado y las prácticas a partir de las cuales se forman aquellas. La dimensión arqueológica del análisis permite analizar las formas mismas de la problematización; su dimensión genealógica, su formación a partir de las prácticas y de sus modificaciones". (Foucault, 2004, pp. 14-15) ${ }^{4}$

El arsenal teórico-metodológico foucaultiano brinda, pues, insumos suficientes y necesarios para el desarrollo de indagaciones particulares, de pesquisas concretas, bien acotadas, de temas y problemas que yacen al margen de las grandes historias y de las macro-teorías; historias de los bordes, de los pliegues, de los quiebres, de las discontinuidades..., que se traman en las prácticas sociales concretas y que permiten localizar la emergencia de nuevas formas de saber, de nuevas relaciones de poder, de nuevas modalidades de sujetos; como señala el mismo Foucault (1970):

por detrás de la historia atropellada de los gobiernos, de las guerras y de las hambres, se dibujan unas historias, casi inmóviles a la mirada, historias de débil declive: historia de las vías marítimas, historia del trigo o de las minas de oro, historia de la sequía y de la irrigación, historia de la rotación de cultivos, historia del equilibrio obtenido por la especie humana, entre el hambre y la proliferación. (p. 4)

La arqueología, entonces, posibilita la realización de descripciones históricas, a base de enunciados y archivos, documentos (monumentos), teniendo en consideración lo efectivamente dicho, hecho o escrito, y para el caso de la indagación propuesta, la realización de una caracterización de las formas de evaluación educativa en los ámbitos y las periodizaciones ya señalados.

4 Cf. Martiarena (1995, pp. 105-106). 
En lo tocante a la genealogía es preciso señalar que esta no puede concebirse como ruptura escindida de la arqueología; al contrario, es necesario entender que arqueología y genealogía se fundamentan en unos supuestos comunes: la descripción de historias sin que sus análisis se refieran a la instancia de un sujeto fundador, trascendente. El giro hacia la genealogía implica, según los señalamientos de Castro (2004), una ampliación del campo investigativo, "para incluir de manera más precisa el estudio de las prácticas no-discursivas y, sobre todo, la relación no-discursividad/discursividad; dicho de otro modo: para analizar el saber que en términos de estrategia y tácticas de poder" (p. 146). Ello supone, por una parte, ubicar el saber sobre ámbitos de lucha, sobre antagonismos, sobre incitaciones recíprocas y reversibles; por la otra, determinar las condiciones de posibilidad para la emergencia, la aparición, la invención de algo (un saber, un objeto, un concepto, una institución, un acontecimiento...), invención que en todo caso se manifiesta a través del enfrentamiento de fuerzas, de poderes, de saberes.

El análisis genealógico (segunda etapa de la indagación) se orienta, entonces, como se refirió en la presentación de la propuesta de Sánchez, Vanegas, Moreno, Rincón, Montenegro y Baena (2008):

a la determinación de las condiciones de posibilidad que han permitido y hecho posible la emergencia e instalación de la maquinaria evaluativa, que opera de diversos modos a la manera de dispositivos de agenciamiento, de promoción, de selección, de cualificación, de gestión, de inclusión, de exclusión, etc. (p. 4) ${ }^{5}$

La indagación acerca de los discursos y las prácticas evaluativas en la USTA finiquitaría con una analítica-interpretativa acerca de las modalidades de sujetos que son configurados a través de la maquinaria panevaluativa, toda vez que estudiantes, docentes, administrativos, directivos, agentes internos y externos de la institución (los integrantes todos de la comunidad académica) son (somos) cifrados, analizados, evaluados, examinados, conducidos, moldeados, formateados, agenciados, gestionados, fabricados, disciplinados,

5 El análisis genealógico para la indagación propuesta se funda en el conjunto de discurso normativosprescriptivos que explicitan "múltiples modalidades evaluativas que ponen en marcha una pluralidad de relaciones que implican diversos escenarios, actores, instancias, procedimientos, acciones, procesos..., que coparticipan de la vida universitaria" (pp. 4-5). 
vigilados, controlados, auto-regulados, auto-evaluados, auto-gestionados, de múltiples maneras dada la puesta en funcionamiento de dichas prácticas.

Esta última -y novedosa por demás- forma de análisis acerca de la omnipresencia de la evaluación, de la adopción de una nueva cultura que todo lo examina, de la extensión por todas partes de un nuevo culto que todo lo juzga, abre una variedad de posibilidades para la empresa de nuevas y posteriores indagaciones históricas, que como se ha referido, yacen invisibles, ocultas, para los grandes relatos. En tales historias vemos, no obstante, que los sujetos, los objetos, los poderes, las instituciones, los saberes mismos, son a la vez constituidos y constituyentes.

Los recortes teórico-metodológicos señalados, en los que se ponen en acción las piezas de la caja de herramientas, constituyen un programa de investigación en que se articula, bajo un perfil arqueológico-genealógicohermenéutico, una ontología histórica de nuestro propio presente en relación con el (un) saber, el poder -o mejor, unas relaciones de poder-y unas modalidades de constitución de sujetos, que subyacen a las prácticas discursivas y no-discursivas referidas a la evaluación. Un símil, salvando las proporciones, de lo que, al decir de Morey, realizó Foucault (1990) en su obra:

[Una] ontología de nosotros mismos en relación a la verdad que nos constituye como sujetos de conocimiento.

[Una] ontología histórica de nosotros mismos en las relaciones de poder que nos constituyen como sujetos actuando sobre los demás.

[Una] ontología histórica de nosotros mismos en la relación ética por medio de la cual nos constituimos como sujetos de acción moral. (p. 25).

En este artículo nos ocupamos fundamentalmente de la herramienta arqueológica, en virtud de que ésta comporta la metodología para el desarrollo de la propuesta investigativa en la primera etapa. 


\section{El recurso arqueológico}

Como se ha referido, la arqueología ofrece la posibilidad de escribir historias generales, elaboradas sobre espacios de dispersión referidas a tematizaciones y problematizaciones específicas, en contraposición a las historias globales que agrupan "todos los fenómenos en torno de un centro único: principio, significación, espíritu, visión del mundo, forma de conjunto" (Foucault, 1970, p. 16). Este tipo de historia -global-, según señala Foucault, ha comenzado a deslindarse a favor del reconocimiento de las condiciones de posibilidad para la emergencia -o las emergencias- de conceptos, objetos, temas, problemas..., que comúnmente se hallan en los márgenes, en los bordes relegados, plegados de lo que las sociedades determinan como discursos válidos. Así las cosas, la arqueología como herramienta metodológica faculta la realización de historias concretas, como la de las formas evaluación educativa en la Universidad Santo Tomás.

La primera de estas herramientas, la arqueología - desarrollada en la primera etapa del pensamiento foucaultiano ${ }^{6}-$, busca determinar las condiciones de posibilidad del saber (Castro, 2004); es un método de análisis de los discursos y de las prácticas, o mejor, de las prácticas discursivas, que pretende investigar el conjunto de reglas generales que determinan las múltiples relaciones que se tejen entre los enunciados constituyentes de un saber determinado, en una época dada. Esta connotación nos ubica en el terreno de la indagación propuesta y nos permite los ajustes o recortes pertinentes:

- El saber constituido por los discursos y las prácticas (referido a las formas evaluación educativa).

6 La primeras búsquedas arqueológicas de Foucault, según los señalamientos de Morey (1983), están contenidas en sus obras iniciales: Historia de la locura, Nacimiento de la Clínica, Las palabras y las cosas, de las cuales La arqueología del saber es un texto balance, un inventario crítico, una reformulación sistematizada de los procedimientos metodológicos expuestos en tales obras. La arqueología del saber es la respuesta de Foucault a un conjunto de cuestionamientos emergentes de sus primeros escritos; en ella señala "sus distancias, y el carácter de 'caja de herramientas' o manual de instrucciones de uso que el texto revela a nivel de su estructura misma" (p. 183); el mismo Foucault sostiene: "Más que fundar en derecho una teoría $\square \mathrm{y}$ antes de poder hacerlo eventualmente (no niego que lamento no haberlo conseguido aún) $\square$, se trata, de momento, de establecer una posibilidad" (1970, p. 194). 
- Que enuncian los diversos modos como ese saber y esas prácticas operan en un espacio social (institucional) específico (la Universidad Santo Tomás, sede Bogotá, modalidad presencial).

- En una periodización perfectamente acotada, desde su refundación (1965) hasta el presente.

Las indagaciones históricas, de corte arqueológico, constituyen una crítica de la concepción trascendental de la historia, que postula la exigencia de un origen y un final de las cosas y se aparta por ello de cualquier forma de teleología y causalismo. En aquellas, se procura una renuncia radical a los conceptos de devenir, evolución y progreso, y se omite el planteamiento de la totalidad como ámbito central en que el actor principal es un macro-sujeto racional (Ceballos, 2000). Estas connotaciones facultan el uso de la herramienta arqueológica toda vez que nos permite definir las reglas de formación, operación y transformación de los discursos; los umbrales que deben sortear en la pretensión de lograr determinados estatutos - pretensiones de cientificidad-; el juego, en fin, de las diferentes prácticas, acciones y/o relaciones que traman entre sí las prácticas mismas y los discursos evaluativos que han acompañado el devenir de la USTA. Todo esto, en contraposición a la noción unitaria y totalizante de un discurso omnicomprensivo, siempre lineal y en permanente desarrollo lógico, verbigracia un concepto de evaluación que, al decir de algunos, es condición natural del hombre-como medida y medidor-, por lo cual ha hecho presencia a lo largo de la historia de la humanidad.

Por otra parte, la herramienta arqueológica es valiosa para este tipo de descripciones históricas ${ }^{7}$, en la medida en que:

- Permite revisar el valor del documento, lo trata como monumento en la perspectiva de determinar la ley de existencia de los enunciados y sus regímenes de coexistencia (con otros enunciados y con otras prácticas discursivas o no discursivas). La arqueología se ocupa de la descripción intrínseca del monumento ${ }^{8}$.

$7 \quad$ Cf. Foucault (1970) y Morey (1983).

8 En este sentido, glosando a Foucault (2004), podemos señalar que hacemos uso para nuestros análisis de documentos (monumentos) de orden prescriptivo "textos que, sea cual fuere su forma (discurso, diálogo, tratado, compilación de preceptos, cartas [códigos, reglamentos, políticas], etc.), su objeto principal es proponer reglas de conducta... textos 'prácticos' que en sí mismos son objeto de 'práctica' en la medida 
- Es un dominio aplicado al saber, no a la ciencia, no al conocimiento como tal, no a las disciplinas o a las transdisciplinas, sino a los saberes, mucho más, a aquellos marginados, ignorados, relegados, desconocidos, sobre los que no se ha hecho historia.

- Establecen para la historia su carácter discontinuo.

- Restituyen al enunciado su carácter de acontecimiento.

- Responden, por tanto, a estas preguntas: ¿por qué este enunciado y no cualquier otro en su lugar? ¿Es posible definir un método de análisis histórico liberado del tema antropológico?

- Reclaman para el saber un dominio autónomo, independiente del sujeto, un análisis histórico escindido, por una parte, del sujeto fundador; pues se nos ha hecho creer que es siempre el sujeto quien crea, produce, representa, transforma, usa... el saber ${ }^{9}$. Foucault (1980) sostiene al respecto:

Actualmente, cuando se hace historia -historia de las ideas, del conocimiento o simplemente historia-, nos atenemos a ese sujeto de conocimiento y de representación como punto de origen a partir del cual es posible el conocimiento y la verdad aparece (1980, p. 14).

Por otra parte, despojado del tema de la historia continua, para pensarla en términos de discontinuidad, bajo la clave de la diferencia y el acon-

en que están hechos para ser leídos, aprendidos, meditados, utilizados, puestos a prueba y en que buscan constituir finalmente el armazón de la conducta diaria... textos [que] tienen como función ser operadores que permitan a los individuos interrogarse sobre su propia conducta, velar por ella, formarla y darse forma a sí mismos como sujetos éticos" (p. 15). La caracterización de las formas evaluación educativa, en especial, su descripción histórica "parte del análisis de los diversos discursos que se han desarrollado en torno de la evaluación educativa y, de las prácticas que se han instalado en la institución; por lo cual, se remite a una masa documental que abarca: las principales Reformas en Políticas Educativas, Lineamientos Curriculares, Documentos correspondientes a Evaluación, Leyes y Decretos; Documentos Institucionales: Estatuto Orgánico de la Universidad, Proyecto Educativo Institucional, Estatuto Docente; teoría y metateoría sobre evaluación; y, los diversos instrumentos de evaluación que se han aplicado y se aplican en la institución. Estos documentos constituyen los archivos fundamentales a partir de los cuales se realiza el análisis descrito" (Sánchez et al., 2006, p. 4).

9 En la primera de las conferencias recogidas en La verdad y las formas jurídicas, antes de plantear la hipótesis sobre las diversas historias de la verdad, Foucault (1980) señala que no solamente el sujeto produce o funda el conocimiento, sino que aquél es también producido por éste, constituido y re-constituido por el conocimiento, y afirma que "sería interesante, que intentáramos ver cómo se produce, a través de la historia, la constitución de un sujeto que no está dado definitivamente, que no es aquello a partir de lo cual la verdad se da en la historia, sino al de un sujeto que se constituyó en el interior mismo de ésta y que, a cada instante, es fundado, y vuelto a fundar por ella... La constitución histórica de un sujeto de conocimiento a través de un discurso tomado como un conjunto de estrategias que forman parte de las prácticas sociales" (p. 14-15). 
tecimiento, en la que son fundamentales conceptos como: umbrales, rupturas, límites, transformaciones (Morey, 1983).

- La arqueología tiene como punto de partida la realización de un trabajo negativo, toda vez que busca "liberarse de todo un juego de nociones que diversifican, cada una a su modo, el tema de la continuidad" (Foucault, 1970, p. 33).

El análisis arqueológico nos permite hacer un recorrido por diversos ejes en constante interrelación: práctica discursiva, saber y disciplina, ejes en los que se pueden determinar unos campos de saber, unos dispositivos de poder, unas instituciones y unos sujetos. En este sentido, la indagación propuesta se pliega a los desarrollos teóricos de Zuluaga (1987), que opta por el modelo metodológico denominado "análisis de los saberes institucionalizados", cuyas instancias metodológicas aplicadas a la pesquisa serían: -saber (es), que constituyen el discurso sobre lo evaluativo; - poder (es), fuerzas y relaciones de poder; -instituciones, en que se han instalado diversas prácticas de evaluación; y -sujetos, (docentes, discentes, administrativos, etc.), que gestionan la evaluación y son a la vez gestionados por ella; todo esto, implicado en una compleja red de relaciones que se tejen entre sí y con los campos en que se desarrollan. Así las cosas, la evaluación puede entenderse como un conjunto de estrategias que gestiona saberes, poderes y subjetividades, por lo cual constituirían

deteminaciones de poder y saber que actúan en un campo complejo y heterogéneo de fuerzas diseminadas en las prácticas sociales (incluyendo entre éstas las prácticas discursivas); tales determinaciones requieren para su despliegue una red de instituciones, sujetos y discursos (saberes), atravesados por sus fines o delimitados para sus fines para inducir mecanismos estables de dominación en el conjunto de las fuerzas. (Zuluaga, 1987, p. 141)

\section{Procedimientos para el abordaje arqueológico}

Tres procedimientos fundamentales constituyen el andamiaje para la puesta en escena de la herramienta arqueológica: la descripción de las regularidades discursivas, la descripción del enunciado y el archivo, y la caracterización propia de la arqueología. 


\section{Descripción de las regularidades discursivas}

La primera tarea de la arqueología consiste en el establecimiento de las regularidades discursivas; para lo cual es preciso determinar la unidad (o las unidades) de análisis que yacen presentes en un conjunto de acontecimientos dispersos; reconocer, no la infinita continuidad del discurso y su presencia tradicional en términos de causa-efecto, sino prestar atención a "cada momento del discurso en su irrupción de acontecimiento" (Foucault, 1970, p. 41).

Esta primera labor nos permite despojarnos de la historia tradicional y de sus nociones de continuidad y totalidad para ubicarnos en un gran dominio conformado, según Foucault (1970), por "el conjunto de todos los enunciados efectivos [hablados o escritos acerca de la evaluación], en su dispersión de acontecimientos y en la instancia que le es propia a cada uno"; con lo cual es posible "una descripción pura de los acontecimientos discursivos ${ }^{10}$ como horizonte para la búsqueda de las unidades que en ellos se forman"(p. 43). La descripción del campo de acontecimientos discursivos plantea una cuestión clave para la arqueología: “¿cómo es que ha aparecido tal enunciado y ningún otro en su lugar"? (p. 44). La determinación de las unidades del discurso demanda dejar en suspenso un conjunto de categorías propias de los grandes discursos (tradición, influencia, desarrollo y evolución, mentalidad y espíritu) que relacionan los discursos, (género, libro, obra) que clasifican los discursos, (origen e interpretación) que garantizan una continuidad infinita (Castro, 2004, p. 93).

Un segundo elemento de esta primera etapa de la descripción arqueológica expone relación con las formaciones discursivas, definidas por Foucault (1970) como "el sistema enunciativo general al que obedece un grupo de actuaciones verbales... [y] a unos sistemas lógico, lingüístico y psicológico" (p. 96). Morey (1983), por su parte, señala: "estamos ante una formación

10 Foucault (1970) define el campo de acontecimientos discursivos como "el conjunto siempre finito y actualmente limitado de las únicas secuencias lingüísticas que han sido formuladas, las cuales pueden muy bien ser innumerables, pueden muy bien, por su masa, sobrepasar toda capacidad de registro, de memoria o de lectura, pero constituyen, no obstante, un conjunto finito" (p. 44), de manera que uno de los propósitos de la arqueología es el análisis del campo discursivo, análisis en que pretende "captar el enunciado en la estrechez y la singularidad de su acontecer; de determinar las condiciones de su existencia, de fijar sus límites de la manera más exacta, de establecer sus correlaciones con los otros enunciados que pueden tener vínculos con él, de mostrar qué otras formas de enunciación excluye"; en últimas, responder a esta pregunta: “¿cuál es, pues, esa singular existencia, que sale a la luz en lo que se dice, y en ninguna otra parte?" (p. 45). 
discursiva si pueden establecerse entre un grupo de enunciados una regularidad" -sistema de dispersión de los enunciados-, bajo dos condiciones: correlación entre el análisis del enunciado y la formación discursiva; pertenencia del enunciado a una formación discursiva (p. 196).

En relación con las formaciones discursivas, Foucault (1970, p. 50-58) plantea cuatro hipótesis fundamentales: 1) "los enunciados diferentes en su forma, dispersos en el tiempo, constituyen un conjunto si se refieren a un solo y mismo objeto"; 2 ) es necesario determinar el carácter constante de la enunciación, su estilo, su forma y tipo de encadenamiento; 3 ) establecer los grupos de enunciados, "determinando el sistema de los conceptos permanentes y coherentes que en ellos se encuentran en juego, sus apariciones $y$ su dispersión"; y 4) determinar la identidad y persistencia de los temas. Estas hipótesis pueden operar sobre cualquier objeto de estudio, en nuestro caso, las prácticas y los discursos evaluativos en una periodización diferenciada.

En todo caso, las formaciones discursivas (descripción de la dispersión de los enunciados, definición de la regularidad de los objetos, los tipos de enunciación, los conceptos y las elecciones temáticas) obedecen a unas reglas determinadas, a "unas condiciones a que están sometidos los elementos de esa repartición (objetos, modalidad de enunciación, conceptos, elecciones temáticas). Las reglas de formación son condiciones de existencia (pero también de coexistencia, de conservación, de modificación y de desaparición) en una repartición discursiva determinada" (Foucault, p. 62). La determinación de tales formaciones discursivas (en su unidad e individualidad) implica algunos elementos, a saber:

En primera instancia, la determinación de la formación de los objetos de un discurso. Es necesario, señala Foucault, "definir esos objetos... refiriéndolos al conjunto de las reglas que permiten formarlos como objetos de un discurso y constituyen así sus condiciones de aparición histórica" (pp. 78-79); se trata, al decir de Morey (1983), "de hacer valer el carácter de acontecimiento que tienen los enunciados que constituyen, en su especificidad de tales, los discursos" (p. 198); de la determinación de los objetos que configuran un discurso dado. Para determinar el régimen de los objetos de un discurso se demandan tres condiciones básicas: 
- Localizar las superficies primeras de emergencia de los objetos (condiciones de posibilidad para la irrupción de discursos y prácticas evaluativas);

- Descubrir sus instancias de delimitación (educación, pedagogía, administración, psicología, psicometría, estandarización, políticas nacionales e internacionales, sistemas educativos-evaluativos...); y

- Analizar las rejillas de especificación ("sistemas según los cuales se separa, se opone, se entronca, se reagrupa, se clasifica, se hacen derivar unas de otras las diferentes [formas evaluativas] como objetos de un discurso [educativo-evaluativo].

Determinadas cada una de estas condiciones, es necesario establecer las relaciones que se tejen entre ellas, para que el análisis en su conjunto permita responder a la cuestión: ¿por qué este objeto y no cualquier otro en su lugar? ${ }^{11}$

En segunda instancia, debe determinarse la formación de las modalidades enunciativas. Con el objeto de "caracterizar el régimen de enunciación de un discurso en su dispersión", es necesario establecer:

- El estatuto del hablante (¿Cuál es el estatuto de los individuos que tienen la posibilidad de pronunciar un discurso cualquiera, referido a la evaluación?;

- Los ámbitos institucionales que circundan al hablante (el sistema educativo, la forma escuela, organizaciones nacionales e internacionales que direccionan los discursos y las prácticas evaluativas); $y$

- La posición de sujeto (posición que le es posible ocupar al individuo: parlante, enunciante, interrogante, crítico, observador, emisor, receptor, sujeto u objeto... de los discursos y las prácticas evaluativas).

El análisis de estos elementos y de sus relaciones permiten responder a cota cuestión capital para la arqueología: ¿por qué ésta enunciación y no otra en su lugar? ${ }^{12}$

11 Cf. Foucault (1970, pp. 85-61) y Morey (1983, pp. 197-199).

12 Cf. Foucault (1970, pp. 82-90) y Morey (1983, pp. 199-200). 
Posteriormente, es necesario determinar la formación de conceptos. A fin de "describir la organización del campo de enunciados en el que aparecen y circulan los conceptos, es pertinente fijar:

- Las formas de sucesión y encadenamiento de los enunciados (ordenaciones de las series enunciativas, tipos de dependencia de los enunciados y esquemas retóricos que permiten la combinación de los enunciados o de los grupos de enunciados);

- Las formas de coexistencia (campo de presencia, enunciados evaluativos formulados en otros lugares y tiempos; campo de concomitancia, enunciados que proceden de otros dominios de saber y que pueden valer como elementos de analogía; dominios de memoria, enunciados que establecen relaciones de filiación, génesis, transformación, continuidad, discontinuidad histórica); $y$

- Los procedimientos de intervención que pueden ser aplicados legítimamente a los enunciados (técnicas de reescritura, métodos de trascripción, modos de traducción, medios para acrecentar la aproximación de los enunciados, maneras de delimitación de la validez de los enunciados, modos como se transfiere un tipo de enunciado de un campo de aplicación a otro, métodos de sistematización de proposiciones que existan separadamente, métodos de redistribución de enunciados ligados, en un nuevo conjunto sistemático $)^{13}$.

Finalmente, es necesario determinar la formación de las estrategias. Foucault (1970) entiende por estrategias diversos discursos con cualquier nivel de formalidad, que "dan lugar a ciertas organizaciones de conceptos, a ciertos agrupamientos de objetos, a ciertos tipos de enunciación, que forman, según su grado de coherencia, de rigor de estabilidad, temas o teorías" (p. 105). Para determinar la formación de estrategias de una formación discursiva, deben identificarse:

- Los puntos de difracción posibles del discurso (puntos de incompatibilidad, puntos de equivalencia, puntos de enganche de una sistematización); 
- Las instancias específicas de decisión, elecciones que se realizan entre diversas posibles (sistemas formales, modelos concretos, relaciones - de analogía, de oposición, de complementariedad-con otros discursos, relaciones de delimitación recíproca), que permiten determinar "las posibilidades discursivas que, en un discurso, se encuentran efectivamente realizadas" (Morey, 1983, p. 206);

- Las relaciones entre discurso y no-discurso (la función que debe ejercer el discurso estudiado en un campo de prácticas no-discursivas; el régimen y los procesos de apropiación del discurso; las posiciones posibles del deseo en relación con el discurso) ${ }^{14}$.

Las formaciones discursivas que describe Foucault (1970, p. 117-127) no son meras yuxtaposiciones, coexistencias o interacciones de elementos heterogéneos (saberes, poderes, sujetos, instituciones, técnicas, grupos sociales, organizaciones perceptivas..., referidas a los discursos y a las prácticas evaluativas), sino su trama de relaciones a través de la práctica discursiva, cuyos niveles (diversos) no son independientes unos de otros. Por otra parte, las formaciones discursivas son móviles, residen en el mismo discurso y no son ajenas al tiempo; se transforman, dependen tanto del juego interno de los distintos niveles que la articulan como de la interrelación entre dos dominios: multiplicidades prácticas enunciativas (discursos), multiplicidades prácticas no-enunciativas (instituciones), de manera que un acontecimiento práctico-institucional reformula el discurso y a la inversa, "las prácticas discursivas modifican los dominios que ponen en relación" (Foucault, p. 124). Esto puede evidenciarse en las diversas reformas que se han dado, a nivel general, de las políticas evaluativas que operan sobre el conjunto del sistema educativo y, a nivel específico, en las prácticas evaluativas que se ponen en funcionamiento en la cotidianidad de la acción educativa.

\section{Descripción del enunciado y el archivo}

La segunda tarea de la descripción arqueológica consiste en la definición del enunciado y del archivo. En relación con el enunciado, Foucault (1970) señala que, a primera vista, se conceptualizaría como el "elemento último,

14 Cf. Foucault (1970, pp. 105-116) y Morey (1983, pp. 204-206). 
que no se puede descomponer, susceptible de ser aislado por sí mismo y capaz de entrar en un juego de relaciones con otros elementos semejantes a él"; el "Átomo del discurso" (p. 133).

Un estudio del enunciado en relación y comparación con la proposición, la frase y los actos de habla, le permite al autor indicar que el enunciado es una unidad idéntica pero diferente a la proposición de los lógicos, a la frase de los gramáticos, a los speech act de los analistas; pero es, de la misma manera, en su modo de ser singular, "indispensable para que se pueda decir si hay o no frase, proposición, acto de lenguaje; y para que se pueda decir si la frase es correcta (o aceptable, o interpretable), si la proposición es legítima y está bien formada, si el acto se ajusta a los requisitos y si ha sido efectuado por completo" (Foucault, p. 144). Así pues, el enunciado es "el soporte o la sustancia accidental: en el análisis lógico, es lo que "queda" cuando se ha extraído y definido la estructura de proposición; para el análisis gramatical, es la serie de elementos lingüísticos en la que se puede reconocer o no la forma de una frase; para el análisis de los actos del lenguaje, aparece como el cuerpo visible en el que estos se manifiestan" (p. 140); y admite, con las respectivas reservas, que "existe enunciado en cuanto existen varios signos yuxtapuestos..., en cuanto existe uno, y sólo uno. El umbral del enunciado sería el umbral de la existencia de los signos" (p. 141). En últimas, el enunciado es definido como:

una función de existencia que pertenece en propiedad a los signos y a partir de la cual se puede decidir, a continuación, por el análisis o la intuición, si "casan" o no, según qué reglas se suceden o se yuxtaponen, de qué con signo, y qué especie de acto se encuentra efectuado por su formulación (oral o escrita)... No es en sí mismo una unidad, sino una función que cruza un dominio de estructuras y de unidades posibles y que las hace aparecer, con contenidos concretos, en el tiempo y en el espacio (p. 145).

El enunciado se caracteriza, entonces, no por ser una unidad de tipo lingüístico, sino por ser una "función enunciativa" que trama con las unidades y con el campo de objetos un conjunto de relaciones; les abre un conjunto de posiciones subjetivas posibles; las coloca en un dominio de coordinación y coexistencia; las aloja en un espacio en el que son aprehendidas, utilizadas y repetidas. Lo que queda al descubierto es "el campo de ejercicio de la fun- 
ción enunciativa y las condiciones según las cuales hace aparecer unidades diversas" (Foucault, p. 198). Más que unidad, el enunciado se define como función: es lo que hace existir a un conjunto de signos (actos verbales, frases, proposiciones) y permite un conjunto de reglas o formas que constituyen la función enunciativa, su actualización, las condiciones de posibilidad para la configuración de un discurso.

El enunciado -o mejor, la función enunciativa- presenta cuatro propiedades básicas:

- El enunciado está ligado a un referencial. "La función enunciativa remite, no a un correlato, (en términos de sentido o verdad), sino a su dominio de posibilidad" (Morey, 1983, p. 191) ${ }^{15}$; no a la validez, a la veracidad, al sentido - de la evaluación, en nuestro caso-, sino a un dominio teóricopráctico en el que emerge, ya como práctica o como discurso.

- El sujeto del enunciado es una función vacía. "La función enunciativa determina la posición de sujeto" (Morey, p. 192). El sujeto de la enunciación no puede concebirse como el autor de la formulación. Si existen enunciados, "no es en la medida en que ha habido, un día, alguien que los profiriera o que dejara en alguna parte su rastro provisorio; es en la medida en que puede ser asignada la posición del sujeto" (Foucault, 1970, p. 159). Se ha dicho que la arqueología no fija su atención en el sujeto sino en lo que efectivamente ha sido dicho o escrito; no quien dice algo acerca de la evaluación, sino qué se ha dicho de ella ${ }^{16}$.

- La función enunciativa presupone la existencia de un dominio asociado, un campo adyacente con el cual forma una trama compleja. Este dominio asociado está constituido por:

15 El enunciado, señala Foucault (1970), "está ligado más bien a un 'referencial' que no está constituido por 'cosas', por 'hechos', por 'realidades', por 'seres', sino por leyes de posibilidades, reglas de existencia para los objetos que en él se encuentran nombrados, designados o descritos, para las relaciones que en él se encuentran afirmadas o negadas. El referencial del enunciado forma el lugar, la condición, el campo de emergencia, la instancia de diferenciación de los individuos o de los objetos, de los estados de cosas y de las relaciones puestas en juego por el enunciado mismo [la evaluación, o las formas evaluativas]; define las posibilidades de aparición y de delimitación de lo que da a la frase su sentido, a la proposición su valor de verdad". (p. 152).

"Describir una formulación en tanto que enunciado -señala Foucault (1970)- no consiste en analizar las relaciones entre el autor y lo que ha dicho (o querido decir, o dicho sin quererlo), sino en determinar cuál es la posición que puede y debe ocupar todo individuo para ser su sujeto" (p. 160). 
la serie de las demás formulaciones en el interior de las cuales el enunciado se inscribe y forma un elemento...; el conjunto de las formulaciones a que el enunciado se refiere (implícitamente o no)...; el conjunto de formulaciones cuyo enunciado prepara la posibilidad ulterior, y que pueden seguirlo como su consecuencia, o su continuidad natural, o su réplica...; el conjunto de formulaciones cuyo estatuto comparte el enunciado en cuestión, entre las cuales toma lugar sin consideración de orden lineal, con las cuales se eclipsará, o con las cuales, por el contrario, se valorizará, se conservará, se sacralizará y se ofrecerá, como objeto posible, a un discurso futuro ${ }^{17}$.

- La función enunciativa debe tener una existencia material. La función enunciativa se caracteriza por ser un régimen de materialidad repetible, constituye una evidencia, "tiene una sustancia, un soporte, un lugar y una fecha"; si estos elementos cambian la identidad de enunciado se modifica (por ejemplo, los discursos evaluativos que emergen se afirman, se confirman o desaparecen, para irrumpir nuevamente bajo otros aspectos: evaluación de contenidos, evaluación por objetivos, indicadores, logros, competencias, estándares, cultura de la evaluación...). Foucault (1970), al respecto, explica:

En lugar de ser una cosa dicha de una vez y para siempre..., el enunciado, a la vez que surge en su materialidad, aparece con un estatuto, entra en unas tramas, se sitúa en unos campos de utilización, se ofrece a traspasos y a modificaciones posibles, se integra en operaciones y en estrategias, donde su identidad se mantiene o se pierde. Así, el enunciado circula, sirve, se sustrae, permite o impide realizar un deseo, es dócil o rebelde a unos intereses, entra en el orden de las contiendas y de las luchas, se convierte en tema de apropiación o de rivalidad ${ }^{18}$. (pp. 167-177)

17 Cf. Foucault (1970, pp. 164-165) y Morey (1983, pp. 192-193). Para el caso de la indagación propuesta, existe todo un dominio asociado, en el cual puede verse emerger el enunciado evaluación o la función enunciativa relativa a los discursos y prácticas evaluativas; dominio asociado que implica: formulaciones en el interior de las cuales el enunciado se inscribe y forma un elemento (educación, sociedad, sistema educativo, sistemas evaluativos...); formulaciones a las que el enunciado se refiere (evaluación, examen, prueba, selección, clasificación, certificación, acreditación, promoción, titulación, validación, homologación...); formulaciones cuyo enunciado prepara la posibilidad ulterior (sistemas de evaluación, sistemas de selección, sistemas de examen, sistema de pruebas...); formulaciones que permiten ubicarlo como objeto posible para un discurso futuro (sistemas de autoevaluación, autorregulación, sistemas de acreditación, sistemas de gestión, registros calificados...). 
Los desarrollos teóricos foucaultianos acerca de la descripción de los enunciados se realiza a partir de dos interrogantes básicos: ¿qué hay que entender por la tarea de describir unos enunciados? y ¿cómo puede esta teoría del enunciado ajustarse al análisis de las formaciones discursivas que había sido esbozado sin ella? (Foucault, p. 179).

Las respuestas al primer cuestionamiento implican tres momentos (Foucault, pp. 179-181). Primero, la fijación del vocabulario:

- Actuación verbal o lingüística: todo conjunto de signos efectivamente producidos a partir de una lengua natural (o artificial);

- Formulación: acto individual o colectivo que hace aparecer, sobre una materia cualquiera y de acuerdo con una forma determinada, ese grupo de signos (acontecimiento siempre localizable según unas coordenadas espacio-temporales);

- Frase o proposición: las unidades que la gramática o la lógica pueden reconocer en un conjunto de signos;

- Enunciado: la modalidad de existencia propia de este conjunto de signos, que le permite ser algo más que: una serie de trazos, una sucesión de marcas sobre una sustancia, un objeto cualquiera fabricado por un ser humano; que le permite estar en relación con un dominio de objetos, prescribir una posición definida a todo sujeto posible, estar situado entre otras actuaciones verbales, estar dotado de una materialidad repetible;

- Discurso: un conjunto de actuaciones verbales, lo producido en cuanto a conjunto de signos; un conjunto de actos de formulación, una serie de frases o de proposiciones. Foucault (1970) explica: "el discurso está constituido por un conjunto de signos, en tanto que éstas son enunciados, y se les puede asignar modalidades particulares de existencia... conjunto de los enunciados que dependen de un mismo sistema de formación" (p. 181); por ejemplo, discurso clínico, económico, discurso de la historia natural, discurso psiquiátrico; el discurso de la evaluación, de sus prácticas, de sus modalidades, etc.;

- Formación discursiva: principio de dispersión y repetición de los enunciados. Foucault lo define como: "sistema enunciativo general al que 
obedece un grupo de actuaciones verbales, sistema que no es el único que rige, ya que obedece además, y según sus otras dimensiones, a unos sistemas lógico, lingüístico y psicológico" (p. 196) ${ }^{19}$; y

- Práctica discursiva: "Un conjunto de reglas anónimas, históricas y siempre determinadas en el tiempo y en el espacio que han definido en una época dada, y para un área social, económica, geográfica o lingüística dada, las condiciones de ejercicio de la función enunciativa" (Foucault, p. 198).

Segundo momento, descripción de las condiciones de existencia de los enunciados, de las condiciones que han dado al enunciado una existencia específica: relación con un dominio de objetos, juego de posiciones posibles para un sujeto, elemento de un campo de coexistencia, materialidad repetible. El enunciado es, pues, lo no visible y lo no oculto.

No oculto porque lo que cuenta en el enunciado es lo efectivamente dicho en su materialidad (sin recurso a ningún no-dicho o ya-dicho que le subyace). No visible, sin embargo, en virtud de su misma transparencia, por la cual percibimos antes el sentido de lo dicho o su valor de verdad, que ese rasgo primero y de superficie: el que algo sea efectivamente dicho".

De modo que la tarea de la arqueología consiste en "hacer visible lo que sólo es invisible porque está demasiado en la superficie de las cosas" (Morey, 1983, p. 93-94) ${ }^{20}$.

El nivel enunciativo, finalmente, se esboza en su misma proximidad, "no porque el enunciado no esté escondido ha de ser visible" (Foucault, 1970, p.

19 Estamos frente a una formación discursiva cualquiera, si se puede establecer entre un grupo de enunciados una regularidad (temporal o conceptual), bajo cuatro condiciones: que el análisis del enunciado y el de la formación se hallen establecidos correlativamente; que el enunciado pertenezca a una formación discursiva determinada; que dependa de ella, que esté constituido por "un número limitado de enunciados para los cuales se puede definir un conjunto de condiciones de existencia; y, que se circunscriba a una práctica discursiva determinada. (Foucault, 1970, pp. 197-198).

20 De este segundo momento, se sigue que el método arqueológico reclama un nivel específico en su análisis, el nivel enunciativo, en tanto que: se le trate en su materialidad, en su carácter de acontecimiento, en su singularidad; se le remita a un referencial formado por las condiciones de posibilidad de las reglas de existencia de los objetos y relaciones que les encuentran designadas y no a un correlato; se desestime la pregunta por quién ha dicho realmente tal enunciado o que quería decir y se opte por determinar el modo específico de posición de sujeto que especifica el enunciado; se determine el espacio de coexistencia enunciativa; se retenga el carácter de materialidad repetible, analizando sus condiciones estrictas de repetición; se atienda a la inscripción de lo que se dice, sin buscar en lo dicho un no-dicho -hermenéutica- o un sobre/entre dicho -formalización- (Morey, p. 194); el análisis enunciativo es, en últimas, un análisis histórico, "que se desarrolla fuera de toda interpretación" (Foucault, 1970, p. 184). 
186); está siempre involucrado en las diversas unidades (frases, proposiciones, actos de habla), de modo que la tarea del arqueólogo, consiste en "tratar de hacer visible, y analizable, esa tan próxima transparencia que constituye el elemento de su posibilidad" (Foucault, p. 189) ${ }^{21}$.

En relación con la segunda pregunta, Foucault señala que su pretensión no fue la de desarrollar "una teoría en el sentido estricto y riguroso del término: la deducción a partir de cierto número de axiomas, de un modelo abstracto aplicable a un número indefinido de descripciones empíricas", sino que procuró mostrar cómo puede organizarse "un dominio del cual se encuentran sometidos a discusión los enunciados, su principio de agrupamientos, las grandes unidades históricas que pueden constituir, y los métodos que permiten describirlas" (p.192-193); teniendo en consideración la correlatividad entre el enunciado y la formación discursiva, la pertenencia y dependencia de aquél respecto de una formación discursiva específica y su circunscripción a una práctica discursiva determinada.

Todo lo enunciado anteriormente permite señalar, siguiendo el plan de la obra de Foucault (1970), que la descripción arqueológica se ocupa de dos nociones mayores: la función enunciativa y la formación discursiva. Analizar la función enunciativa y la formación discursiva es establecer una positividad; es "tratar un conjunto de actuaciones verbales al nivel de los enunciados y de la forma de positividad ${ }^{22}$ que los caracteriza...; es definir el tipo de positividad de un discurso" (p. 212).

Para abordar el análisis enunciativo y de las formaciones discursivas, es necesario considerar tres tipos de efectos (leyes) que comportan los enunciados: rareza, exterioridad y acumulación.

21 Sin forzar la interpretación, la tarea arqueológica consiste en percibir ocultas bajo diversas conceptualizaciones (examen, prueba, selección...) formas y prácticas de evaluación que circulan con diferentes denominaciones.

22 La positividad de cualquier discurso (historia natural, economía política, medicina clínica, evaluación...) designa su unidad a través del tiempo, más allá de las obras individuales, de los libros, de las obras, de los textos; puede considerarse como el a priori histórico, condición de realidad para unos enunciados, para una historia dada, "que es la de las cosas efectivamente dichas" (p. 216). 
- Rareza, en oposición a la búsqueda de la totalidad. El análisis de los enunciados y de las formaciones discursivas determina el principio "según el cual han podido aparecer los únicos conjuntos significantes que han sido enunciados" (Foucault, p. 201). La ley de la rareza que comporta varios principios: jamás se ha dicho todo (principio de rarefacción); los enunciado se estudian en el límite que los separa de lo que no se ha dicho, en la instancia que los hace surgir con exclusión de todos los demás; no vincula esas exclusiones a una represión; se recogen en totalidades que los unifican (pp. 201-203).

- Exterioridad, en oposición al fundamento trascendental. La descripción histórica está atravesada por la oposición interior-exterior y por la lucha de volver al exterior (o al interior). Esta oposición supone que el campo de los enunciados acepte el lugar de acontecimientos, de regularidades, de entradas en relación, de modificaciones determinadas, de transformaciones sistemáticas; que describa como un campo anónimo cuya configuración define el lugar posible de los sujetos parlantes; que en sus transformaciones y series sucesivas no obedezca a la temporalidad de la conciencia como a su modelo necesario. No se trata de quien habla, sino de lo que dice, en el juego de la exterioridad (Foucault, p. 204-208).

- Acumulación, en oposición a la búsqueda del origen, lo que implica dejar en suspenso conceptos como lectura, rastro, desciframiento, memoria, que definen el sistema que permite revivir el discurso pasado y encontrar su vivacidad perdida; "buscar qué modo de existencia puede caracterizar a los enunciados independientemente de su enunciación" (Foucault, p. 209). La ley de la acumulación supone el análisis de los enunciados en su remanencia; que lo dicho permanezca en el campo de la memoria; que se traten en su forma de aditividad; que se tome en consideración la recurrencia; Foucault afirma: "todo enunciado tiene un campo de elementos antecedentes con relación a los cuales se sitúa, pero tiene el poder de reorganizarlo y redistribuirlo según relaciones nuevas" (p. 211).

La positividad de un discurso, al decir de Foucault, desempeña el papel del $a$ priori histórico; es condición de realidad para unos enunciados; constituye las condiciones de posibilidad de los discursos en su materialidad de sucesos. Es histórico porque determina la modalidad de la existencia de los discursos en su singularidad de acontecimientos (condición de realidad, no de validez); 
en tanto condición de posibilidad del acontecer de los discursos en la historia, está sujeto a avatares, sucesiones y rupturas que constituyen la historia misma. El a priori se define, en síntesis, como el "conjunto de las reglas que caracteriza una práctica discursiva..., no es solamente el sistema de una dispersión temporal; el mismo es un conjunto transformable" (Foucault, p. 217).

Desde la perspectiva arqueológica, los enunciados son, a la vez, acontecimientos (con sus condiciones y su dominio de aparición) y cosas (comportando su posibilidad y su campo de utilización; Foucault, p. 218). A este sistema de enunciados (acontecimientos y cosas articuladas según sus $a$ priori históricos, Foucault denomina "archivo"; archivo es, el sistema general de enunciabilidad y de funcionamiento de los enunciados" (Morey, 1983, p. 214); es, según señala Foucault (1970), "el sistema general de la formación y la transformación de los enunciados" (p. 221), el dominio específico de cuya descripción se ocupa la arqueología. Dice Foucault:

La actualización jamás acabada, jamás íntegramente adquirida del archivo, forma el horizonte general al cual pertenecen la descripción de las formaciones discursivas, el análisis de las positividades, la fijación del campo enunciativo. El derecho de las palabras... autoriza, pues, a dar a todas estas investigaciones el título de arqueología. Este término no incita la búsqueda de ningún comienzo; no emparenta el análisis con ninguna excavación o sondeo geológico. Designa el tema general de una descripción que interroga lo ya dicho al nivel de su existencia: de la función enunciativa que se ejerce en él, de la formación discursiva a que pertenece, del sistema general de archivo del que depende. La arqueología describe los discursos como prácticas especificadas en el elemento del archivo (p. 223).

\section{Descripción arqueológica}

Finalmente, la tercera tarea de la herramienta metodológica consiste en la descripción arqueológica. El punto de partida de esta descripción es la caracterización y diferenciación de la arqueología en relación con la historia de las ideas. Esta se rige por un modelo representativo que pone en escena los acontecimientos según unos principios de reconocimiento; la arqueología adopta el saber y trata de determinar las leyes de su funcionamiento; es 
"abandono de la historia de las ideas, rechazo sistemático de sus postulados y de sus procedimientos, tentativa para ser una historia distinta de lo que los hombres han dicho" (Foucault, p. 232-233). En relación con los temas genéricos característicos de la historia de las ideas (génesis, continuidad, totalización), la arqueología propone cuatro principios que le permiten desmarcarse del análisis tradicional23:

- Tratar los discursos como monumentos y no como documentos, no tiene por finalidad interpretar, no busca"otro discurso" que esté más escondido, que se halle oculto; trata los discursos"en tanto prácticas que obedecen a unas reglas [determinadas]" (Foucault, p. 233).

- No trata de encontrar la transición del discurso con lo que le sigue, precede o rodea, sino definir su especificidad: es "un análisis diferencial de las modalidades de discursos" (Foucault, p. 234).

- No tiene en cuenta el autor sino las prácticas discursivas que atraviesan las obras individuales: "la estancia de sujeto creador en tanto qué razón de ser de una obra y principio de su unidad le es ajena" (p. 235).

- No busca lo que fue querido, pensado o experimentado por los hombres que lo escribieron:"no es nada más y ninguna otra cosa que una reescritura; es decir, una transformación pautada de lo que ha sido y ha escrito. No es la vuelta al secreto mismo del origen, es la descripción sistemática de un discurso-objeto" (p. 235).

Siguiendo los principios referidos, se pueden identificar cuatro puntos básicos en los que la arqueología toma distancia de la historia de las ideas:

- Problema de la asignación de novedad (descripción de la originalidad, estableciendo regularidades de enunciados vs. búsqueda del origen absoluto).

- Análisis de las contradicciones (determinar espacios de disensión de las contradicciones vs. principios de coherencia; supresión de contradicciones). 
- Descripciones comparativas (repartición de la diversidad de discursos en figuras distintas vs. reducción de la diversidad de los discursos; dibuja la unidad que los totaliza).

- Localización de las transformaciones (analiza el cambio mediante la descripción de las transformaciones de los discursos a diferentes niveles y según sus modos específicos vs. sucesiones, encadenamiento primero e indisoluble al que está sometido el discurso por la ley de su finitud (Foucault, pp. 232-297) ${ }^{24}$.

Lo referido muestra, según los señalamientos de Morey (1983), que "el objeto de la arqueología es el análisis y descripción de formaciones discursivas" (p. 221). Estas "no pueden identificarse con disciplinas establecidas: no son ciencias, ni para-ciencias, ni siquiera pre-ciencias, aunque formen el suelo-lo previo- de dónde surgen las ciencias. Es en este sentido que la arqueología se caracteriza como arqueología del saber" (p. 221), entendiendo por saber "ese conjunto de elementos formados de manera regular por una práctica discursiva y que son indispensables a la constitución de una ciencia, aunque no estén necesariamente destinados a darle lugar" (Foucault, 1970, p. 306).

Un saber -señalan los análisis foucaultianos- es aquello de lo que se puede hablar en una práctica discursiva que así se encuentra especificada: el dominio constituido por los diferentes objetos que adquirirán o no un estatuto científico [el saber sobre la evaluación]; un saber es también el espacio en que el sujeto puede tomar posición para hablar de los objetos de que trata en su discurso [saber de quién profiere un discurso relativo a la evaluación]; un saber es el campo de coordinación y de subordinación de los enunciados en que los conceptos aparecen, se definen, se aplican y se transforman [el saber que refiere a los desplazamientos de los sujetos, los objetos, las cosas, las instituciones, los ámbitos..., en los que opera de diversos modos la evaluación]; en fin, un saber se define por posibilidades de utilización y de apropiación ofrecidas por el discurso [usos sociales de la evaluación]. Existen saberes que son independientes de las ciencias..., pero no existe saber sin una práctica discursiva definida; y toda práctica discursiva puede definirse por el saber que forma" (pp. 306-307). 
Entonces, el dominio específico que la arqueología reclama como propio es el saber, desmarcado del estatuto de la ciencia, el saber al que Foucault alude en la hipótesis que formula en la primera conferencia de La verdad y las formas jurídicas:

en realidad hay dos historias de la verdad. La primera es una especie de historia interna de la verdad, que se corrige partiendo de sus propios principios de regulación: es la historia de la verdad tal como se hace en o a partir de la historia de las ciencias. Por otra parte, creo que en la sociedad, o al menos en nuestras sociedades, hay otros sitios en los que se forma la verdad, allí donde se define un cierto número de reglas de juego a partir de las cuales vemos nacer ciertas formas de subjetividad, dominios de objeto y tipos de saber. Por consiguiente, podemos hacer a partir de ello una historia externa, exterior de la verdad (1980, p. 15).

Según Morey (1983, p. 221), para determinar el ámbito propio de la arqueología, Foucault propone "una caracterización de los diferentes regímenes de epistemologización de las formaciones discursivas -sucesión de umbrales posibles que no son ni obligados..., ni cronológicamente sucesivos..., ni cronológicamente homogéneos para las diversas ciencias o disciplinas con pretensiones científicas"; acto seguido, esquematiza dichos regímenes en la Tabla 1.

En últimas, la arqueología se ocupa de"determinar las condiciones de posibilidad de la constitución de determinadas figuras epistemológicas..., sobre el suelo de un saber que les brinda las formas de su positividad" (Morey, 1983, p. 222); intenta dejar al desnudo "las prácticas discursivas en la medida en que dan lugar a un saber y en que ese saber toma el estatuto y el papel de ciencia" (Foucault, 1970, p. 321). 


\section{Tabla 1}

\begin{tabular}{|c|c|c|c|}
\hline $\begin{array}{l}\text { UMBRALES DE } \\
\text { LAS FORMACIO- } \\
\text { NES DISCURSIVAS }\end{array}$ & DEFINICIÓN & $\begin{array}{l}\text { FIGURA DE LA } \\
\text { FORMACIÓN } \\
\text { DISCURSIVA }\end{array}$ & $\begin{array}{l}\text { MODALIDAD } \\
\text { DE HISTORIA DE } \\
\text { LAS CIENCIAS }\end{array}$ \\
\hline $\begin{array}{l}\text { Umbral } \\
\text { de positividad }\end{array}$ & $\begin{array}{l}\text { Momento a partir del que } \\
\text { una práctica discursiva se } \\
\text { individualiza, bajo un único } \\
\text { sistema de formación de los } \\
\text { enunciados. }\end{array}$ & Saber & $\begin{array}{l}\text { Historia } \\
\text { arqueológica }\end{array}$ \\
\hline $\begin{array}{l}\text { Umbral de } \\
\text { epistemolo- } \\
\text { gización }\end{array}$ & $\begin{array}{l}\text { Cuando un conjunto de } \\
\text { enunciados se recorta y } \\
\text { ejerce respecto al saber una } \\
\text { función dominante (verifica- } \\
\text { ción y coherencia). }\end{array}$ & $\begin{array}{l}\text { Figura episte- } \\
\text { mológica }\end{array}$ & $\begin{array}{l}\text { Historia } \\
\text { arqueológica }\end{array}$ \\
\hline $\begin{array}{l}\text { Umbral } \\
\text { de cientificidad }\end{array}$ & $\begin{array}{l}\text { Cuando la figura episte- } \\
\text { mológica obedece no solo } \\
\text { a reglas arqueológicas de } \\
\text { formación, sino a leyes de } \\
\text { construcción de las propo- } \\
\text { siciones (criterios formales). }\end{array}$ & Ciencia & $\begin{array}{l}\text { Historia } \\
\text { epistemológica } \\
\text { de las ciencias }\end{array}$ \\
\hline $\begin{array}{l}\text { Umbral de } \\
\text { formalización }\end{array}$ & $\begin{array}{l}\text { Cuando el discurso científico } \\
\text { puede plantar y desplegar a } \\
\text { partir de sí mismo el edificio } \\
\text { formal que lo constituye. }\end{array}$ & $\begin{array}{l}\text { Ciencia } \\
\text { formal }\end{array}$ & $\begin{array}{l}\text { Análisis } \\
\text { recurrencial }\end{array}$ \\
\hline
\end{tabular}

Nota. De Lectura de Foucault (p. 222), por M. Morey, 1983, Madrid, España: Taurus.

Una de las caracterizaciones de la arqueología es el concepto de episteme, que "entendido como'el análisis de las formaciones discursivas, de las positividades y del saber en sus relaciones con las figuras epistemológicas y las ciencias [y que se diferencia] de las demás formas posibles de historia de las ciencias..." (Foucault, 1970, p. 322). La episteme no comporta un tipo específico de conocimiento o de racionalidad referida a alguna ciencia; tampoco manifiesta la unidad y la soberanía del sujeto fundador, del espíritu o de una época determinada (Morey, 1983). 
Por episteme -Foucault entiende- el conjunto de las relaciones que pueden unir, en una época determinada, las prácticas discursivas que dan lugar a unas figuras epistemológicas, a unas ciencias, eventualmente a unos sistemas formalizados; el modo según el cual cada una de esas formaciones discursivas se sitúan y se operan los pasos a la epistemologización, a la cientificidad, a la formalización...; es el conjunto de las relaciones que se pueden descubrir, para una época dada, entre las ciencias cuando se las analiza el nivel de las regularidades discursivas (p. 223).

Según Castro (2004), la episteme es la figura discursiva que define el campo de descripción de la arqueología, figura que es complementada por otras nociones como: formación discursiva, enunciado, archivo, estrategia..., que constituyen un campo de saber teórico-práctico; en una determinación temporal y geográfica concreta, "que describe la región intermedia entre los códigos fundamentales de la cultura: los que rigen su lenguaje, sus esquemas perceptivos, sus intercambios, sus técnicas, sus valores, la jerarquía de sus prácticas y las teorías científicas y filosóficas que explican todas esas formas del orden" (p. 111); describe, asimismo, las condiciones de posibilidad de los conocimientos, no el punto de vista de su racionalidad o de su objetividad.

El análisis de la episteme (de las formas -discursos y prácticas- evaluativas en la Universidad Santo Tomás) en tanto conjunto de relaciones que pueden unir en una época dada (desde su refundación hasta el presente) las prácticas discursivas (prácticas evaluativas) que pueden dar lugar a determinadas figuras epistemológicas, a disciplinas o saberes con pretensiones de cientificidad, constituye las condiciones de posibilidad de la descripción histórica de esta empresa -primera etapa- en que nos hemos aventurado.

\section{A modo de conclusión}

La propuesta teórica de Foucault abre múltiples posibilidades para diversos procedimientos de investigación señalando una pluralidad de rutas teóricas, epistemológicas, metodológicas, que a la manera de una caja de herramientas, pueden ser usadas, re-utilizadas, re-inventadas..., de diversas 
maneras ${ }^{25}$; así por ejemplo, para la realización de una descripción histórica de una temática determinada, de un campo de saber dado, de un conjunto de teorías específicas, de un discurso o de una práctica discursiva (referidos a la evaluación), se dispone de la arqueología, la cual permite historias singulares, diferenciales, concretas, particulares, que por tales circunstancias no dejan de tener -sin que se persigan como objeto fundamental- condiciones de universalidad, de validez y de objetividad.

Más allá de esta herramienta, los desarrollos teóricos foucaultianos ponen a disposición de la investigación la herramienta genealógica, mediante la cual es posible determinar las condiciones de emergencia, de irrupción, de aparición de un fenómeno determinado, de un objeto dado, de un saber, de una disciplina, de una ciencia; de un poder o de un conjunto de juegos y relaciones de poder. Estas herramientas se complementan con los procedimientos mediante los cuales los sujetos pueden gestionarse o autogestionarse, en la perspectiva de constituirse en sujetos de sí, en sujeto morales; en sujetos que, mediante un conjunto de técnicas y/o tecnologías de subjetividad, se convierten a sí mismos en "auténticas obras de arte". Estos dos dispositivos de análisis que se ponen en escena en las subsiguientes etapas de la pesquisa serán objeto de próximas elaboraciones.

La propuesta investigativa planteada -en su primera etapa-: "Caracterización de las formas evaluación educativa en la Universidad Santo Tomás, Sede Central, Modalidad Presencial, desde su refundación hasta el presente", hace uso de la primera de estas herramientas -la arqueología- que se ocupa de la descripción pura de los enunciados que en su dispersión, en su regularidad, en su singularidad, en su rareza, en su exterioridad...; modalidades enunciativas que constituyen los nodos, los núcleos de los saberes, de las disciplinas, de todas aquellas formas de enunciar, de decir, de nombrar objetos de diversos discursos.

25 A propósito del uso de la "caja de herramientas", se pueden seguir los postulados de Guyot, Marincevic y Becerra (1996), quienes sostienen: "no nos [interesa] repetir a Foucault, ni rebatir sus posiciones, sino usarlo, como él mismo nos enseñó, bajo la presión de los problemas específicos, para saber si es posible construir una nueva política de verdad. Puesto que la única marca de reconocimiento que se puede testimoniar a un pensamiento... es precisamente utilizarlo, deformarlo, hacerlo chirriar, gritar, es que decidimos tomar lo heredado para hacerlo propio" (p. 16). 
La arqueología brinda, pues, la posibilidad de realizar historias particulares en torno de un objeto determinado. Así como Foucault hizo historia de la locura, de la sexualidad, de la clínica, de las prácticas médicas, de las prácticas de vigilancia, control y normalización, de las prácticas de vigilancia y castigo, de las prácticas mediante las cuales los sujetos se constituyen, como ya se dijo, en sujetos morales; concita el abordaje de historias que se traman en las prácticas sociales y que escapan a los análisis de los macro-relatos y de las grandes teorías.

Lo consignado en este ejercicio no es más que una escueta aproximación a los elementos focales de una arqueología, una síntesis que permite un acercamiento, nunca acabado, a una obra que muestra otra forma de hacer historia, otra forma de indagar, otro modo de investigar, otra perspectiva que puede permitir, además, ver las cosas que no se ven, porque están demasiado inmediato de la superficie.

\section{Referencias}

Castro, E. (2004). El vocabulario de Michel Foucault. Buenos Aires, Argentina: Prometeo-Universidad Nacional de Quilmes.

Ceballos, H. (2000). Foucault y el poder. México: Coyoacán.

Foucault, M. (1970). La arqueología del saber (4a. ed). México: Siglo XXI.

Foucault, M. (1980). La verdad y las formas jurídicas. Barcelona, España: Gedisa.

Foucault, M. (1990). Tecnologías del yo y otros textos afines. Barcelona, España: Paidós Ibérica.

Foucault, M. (2004). Historia de la sexualidad. El uso de los placeres (Vol. 2). Buenos Aires, Argentina: Siglo XXI.

Guyot, V., Marincevic, J. y Becerra, M. (1996). Los usos de Foucault. Buenos Aires, Argentina: El Francotirador. 
Martiarena, O. (1995). Michel Foucault: historiador de la subjetividad. México: ITEMS-El Equilibrista.

Morey, M. (1983). Lectura de Foucault. Madrid, España: Taurus.

Sánchez, T., Vanegas, L., Moreno, C., Rincón, I., Montenegro. S. y Baena, S. (2006). Proyecto "Caracterización de las formas evaluación educativa en la Universidad Santo Tomás Sede Bogotá, Modalidad Presencial". Etapa I: Perspectiva Arqueológica. Manuscrito no publicado, Universidad Santo Tomás en Bogotá, Colombia.

Sánchez, T., Vanegas, L., Moreno, C. y Díaz, I. (2008). Proyecto "Caracterización de las formas evaluación educativa en la Universidad Santo Tomás Sede Bogotá, Modalidad Presencial."Etapa ll: Perspectiva Genealógica. Manuscrito no publicado, Universidad Santo Tomás en Bogotá, Colombia.

Zuluaga, O. L. (1987). Pedagogía e historia. La historicidad de la pedagogía. La enseñanza, un objeto de saber. Bogotá, Colombia: Foro Nacional por Colombia. 